\title{
PREDICTORES DEL MIEDO Y LAS HABILIDADES SOCIALES EN ADOLESCENTES DEL CONTEXTO PLURICULTURAL DE CEUTA
}

\section{PREDICTORS OF FEAR AND SOCIAL SKILLS IN ADOLESCENTS IN THE MULTICULTURAL CONTEXT OF CEUTA}

\author{
Federico PUlido-Acosta ${ }^{1}$ y Franciso Herrera-Clavero ${ }^{1}$
}

Cómo referenciar este artículo/How to reference this article:

Pulido-Acosta, F., y Herrera-Clavero, F. (2016). Predictores del miedo y las habilidades sociales en adolescentes del contexto pluricultural de Ceuta [Predictors of fear and social skills in adolescents in the multicultural context of Ceuta]. Acción Psicológica, 13(1), 179-190. http://dx.doi.org/10.5944/ap.13.1.15560

\section{Resumen}

El objetivo fundamental perseguido en este trabajo fue el de conocer los predictores del Miedo y las Habilidades Sociales, así como las relaciones existentes entre ambos de alumnos de 12 a 18 años, en la ciudad de Ceuta. Para ello se contó con 557 participantes, de 4 institutos distintos, que reflejan las características del contexto pluricultural de la ciudad. De ellos el $54.2 \%$ fueron chicas, mientras que el $45.8 \%$ fueron varones. El $64.5 \%$ pertenecían a la cultura/religión musulmana, mientras que el $35.5 \%$ eran cristianos. Como instrumentos de evaluación se emplearon la adaptación de Ascensio, Vila, Robles-García, Páez, Fresán y Vázquez (2012) del Inventario de Miedo para Niños (FSSC-II), junto con la EHS (Escala de Habilidades Sociales, Gismero, 2000).
Los resultados reflejan niveles medio-bajos de Miedo y medio-altos en las Habilidades Sociales. Las Habilidades Sociales sólo se encuentran bajo la influencia de las variables sociodemográficas Género y Cultura. En el Miedo los predictores son el Género y la Cultura/Religión. Existe relación entre Miedo y las Habilidades Sociales, aumentado estas últimas a medida que descienden las puntuaciones en Miedo.

Palabras clave: miedo; habilidades sociales; contexto pluricultural.

\begin{abstract}
The main objective of this paper is to reflect the predictors of Fear and Social Skills, and the influence over each other, of teenagers from 12 to 18 in the city of Ceuta. To make it possible we focused on 557
\end{abstract}

Correspondencia: Francisco Herrera Clavero. Departamento de Psicología Evolutiva y de la Educación, Universidad de Granada.

Email: fherrera@ugr.es

${ }^{1}$ Universidad de Granada, España.

Recibido: 05 de febrero de 2016.

Aceptado: 22 de marzo de 2016. 
participants from 4 different high schools that clearly reflect the city's pluricultural features; $45.8 \%$ of them are boys and $54.2 \%$ are girls; $64.5 \%$ are Muslims and $35.5 \%$ are Christians. The techniques used in this survey are Fear Survey Schedule for Children (FSSC-II), adapted for its use in the Spanish language by Ascensio et al. (2012) as well as the EHS (Gismero, 2000). The results show medium-low levels on Fear and medium-high levels on Social Skills. The Social Skills only are influenced by the sociodemographic factors of gender and custom/religion. The Fear is influenced only by gender and customs/religion. We have also found a relationship between Fear and Social Skills.

Keywords: fear; social skills; pluricultural context.

\section{Introducción}

Los cambios demográficos de las últimas décadas han generado cambios importantes en las relaciones generacionales, pasando de una vida familiar multigeneracional a una intergeneracional, con importantes consecuencias para las relaciones de ayuda y apoyo (Braz, Cômodo, Del Prette, Del Prette y Fontaine, 2013). La actuación social competente puede ser decisiva para el éxito social y profesional. El comportamiento incompetente, en este sentido, posee una serie de consecuencias negativas como el rechazo social, desajustes psicológicos, problemas emocionales y académicos y comportamiento antisocial (Bueno, Durán y Garrido, 2013). Del mismo modo, los estilos adecuados pueden calmar la ansiedad y el estrés, dado que la mayoría de numerosas fuentes de estrés dependen de las relaciones interpersonales. Esto es una importante razón que puede explicar el desarrollo y aceptación de los entrenamientos en habilidades sociales, cuyo objetivo último no es otro que la mejora de la calidad de vida y bienestar de las personas (Delgado, Inglés y García, 2014). De esta forma, se aproximan dos elementos que se consideran en el presente estudios: las emociones de carácter negativo y las habilidades sociales.
Se pone el foco, en una de estas emociones: el miedo, considerada como una emoción específica básica. Como tal, incorpora los componentes fundamentales de ésta. Tiene especial relevancia el componente adaptativo, esencial para la supervivencia de la especie. Otra de las características fundamentales es que se trata de una reacción negativa o de carácter desagradable. Así, el miedo es una emoción de marcado carácter negativo, que se inicia en el procesamiento de estímulos, desencadenando una cascada fisiológica y conductual y con una finalidad adaptativa (Pulido y Herrera, 2015). Los fenómenos causantes de esta emoción tienen escasa variación si se consideran algunos de los factores que se tendrán en cuenta en el presente trabajo. Es lo que ocurre si se consideran género, edad, grupo cultural o estatus socioeconómico, dado que todos pertenecen a la categoría de miedo a la muerte (Ford, Mauss, Troy, Smolen y Hankin, 2014).

La edad ejerce una importante influencia tanto en intensidad como en número de miedos, descendiendo ambos a medida que aumentamos la edad (Kushnir, Gothelf y Sadeh, 2014; Miloyan, Bulley, Pachana y Byrne, 2014; Pulido y Herrera, 2015). No obstante, los niveles permanecen sin excesivas variaciones si nos movemos dentro de una determinada etapa evolutiva, desde la entrada en el periodo de la adolescencia hasta la llegada a la edad adulta (Kushnir et al., 2014; Miloyan et al., 2014). Otro de los factores más relevantes dentro de este campo, es la enorme influencia existente si se considera la variable género. En todos los casos, son las participantes del género femenino las que obtienen niveles más elevados de Miedo (Matesanz, 2006; Pulido y Herrera, 2015). Considerando el entorno cultural y social en el que se realizó el trabajo, se puede remarcar una importante influencia del entorno cultural, social y socio-económico sobre la emoción que se está tratando. Así se describen diferencias estadísticamente significativas considerando estos factores en trabajos como los de Roth et al. (2014) y Pulido y Herrera (2015). Esto hace necesarios instrumentos validados transculturalmente, con poblaciones de distintos contextos lingüísticos y culturales, tal y como se recoge en Tobías-Imbernón, OlivaresOlivares y Olivares (2013).

Las Habilidades Sociales, consideradas como capacidad para manejar el repertorio afectivo y relacio- 
narse con los demás, están muy vinculadas con las capacidades emocionales del sujeto. Del mismo modo que lo observado en la primera variable se puede considerar en el caso de las Habilidades Sociales, mejorando su ejecución a lo largo del desarrollo del sujeto (edad), variando en función del género, en este caso favorable a los varones y también en función de la cultura (Pulido y Herrera, 2015).

Siguiendo a Ford et al. (2014) se pueden marcar relaciones entre emociones negativas y estados sociales y emocionales. Ambas variables pueden llegar a ser inversamente proporcionales tal y como demuestran Pulido y Herrera (2015).

Considerando todo lo mencionado, se presenta el estudio correlacional llevado a cabo con una muestra de adolescentes escolarizados en institutos de la ciudad de Ceuta. Sus objetivos han sido analizar cuáles son los predictores de Miedo y Habilidades Sociales en este alumnado. Como variables se consideraron el género, la edad, la cultura y el estatus socio-económico y cultural. En segundo lugar, se busca conocer qué relación existe entre el Miedo y las Habilidades Sociales. De esta manera se determinará si, cada una de ellas, actúan como predictores de la otra. En base a la revisión bibliográfica realizada se esperan resultados superiores en los niveles de Miedo en función del Género, la Cultura y el Estatus socio-económico. También se esperan diferencias significativas en las puntuaciones obtenidas a través del cuestionario para evaluar las Habilidades Sociales en función de Género y Cultura. Se espera que el Miedo y la Habilidades Sociales sean inversamente proporcionales.

\section{Método}

\section{Participantes}

Para llevar a cabo esta investigación se seleccionó una muestra integrada por 557 participantes repartidos entre cuatro institutos. Por edad, la muestra se distribuye entre el $17.1 \%$ de primero de ESO, el $15.4 \%$ de segundo, el $20.3 \%$ de alumnos de tercero y el $21.5 \%$ de cuarto de ESO. Correspondientes a los Bachilleratos se alcanzan el $12.9 \% \quad$ y el $12.7 \%$ para primero y segundo respectivamente. Considerando la cultura (que se corresponde con la religión), el grupo mayoritario es el de participantes pertenecientes a la cultura-religión musulmana. Estos constituyen el $64.5 \%$ de la muestra. E1 $35.5 \%$ eran cristianos. En función de la variable género, se dan porcentajes muy parecidos, siendo el $45.8 \%$ chicos y el resto $(54.2 \%)$ chicas. Atendiendo al estatus, sólo el $6.3 \%$ de la muestra identificó su nivel como bajo. Fueron menos los que se identificaron como pertenecientes a un nivel alto $(4.8 \%)$. El $38.1 \%$ corresponde al estatus medio-bajo y el $50.8 \%$ al medio. Los participantes se seleccionaron por el método de muestreo incidental o casual. El error muestral fue del $3 \%$.

\section{Instrumentos de evaluación}

Como instrumentos para evaluar el miedo se empleó el Inventario de Miedos para Niños (FSSC-II), en su adaptación al español. Su fiabilidad, en el presente estudio queda reflejada por un alpha de Cronbach de .957. Para la clasificación de los ítems por factores, se obtuvieron los 5especificados en el cuestionario original. Los ítems quedan agrupados de la siguiente forma: dentro del primer factor quedan encuadrados los miedos provocados por lo desconocido y lo extraño. El segundo factor obtenido presenta se relaciona con el miedo a la muerte. El tercero, incluye aquellos ítems vinculados a animales y peligros. Los dos últimos tienen un peso menor y se relacionan respectivamente con los miedos médicos y con miedos desencadenados por temas 
referidos a la escuela (5 ítems). Todos ellos dan una varianza total explicada del $40.07 \%$.

La evaluación de las Habilidades Sociales se hizo a través de la EHS (Gismero, 2000). Su fiabilidad en el presente trabajo fue de .813 . Los factores encontrados fueron 6. El primero (con una varianza explicada de $7.72 \%$ ) se relacionan con la expresión de disconformidad, el segundo con la manifestación de enfado $(7.58 \%)$, el tercero son 4 y se relacionan con la interacción con personas del sexo opuesto $(5.87 \%)$. Para el cuarto subfactor aparecen 5 ítems, relativos a la manifestación de opiniones $(5.83 \%)$. La asertividad corresponde a la quinta categoría $(5.32 \%$ ) y el último subfactor, constituido sólo por 2 ítems (4.5\%) se relaciona con la realización de peticiones. Todo ello da una varianza explicada total del $36.85 \%$.

\section{Procedimiento}

Se solicitaron y obtuvieron las pertinentes autorizaciones, tanto en la Dirección Provincial de Educación, como en cada uno de los diferentes centros. Se respetó escrupulosamente la confidencialidad. Los alumnos, voluntariamente, contestaron los cuestionarios. Todos los cuestionarios fueron pasados por el investigador, en los diferentes centros educativos, en presencia del profesor de cada aula.

Se eligió el método no experimental "ex post facto". Una vez construida la base de datos, se comenzó por el análisis descriptivo, donde se destacaron los porcentajes alcanzados de manera general y en función de las variables sociodemográficas, a través de tablas de contingencia. Con la intención de comprobar los predictores se llevaron a cabo regresiones múltiples paso a paso o "stepwise". Se consideraron como variables criterio las dos variables de estudio comentadas en el apartado anterior: Miedo y Habilidades Sociales. Para hacer el análisis más específico se consideraron también cada uno de los factores que forman parte del Miedo y las Habilidades Sociales, obtenidos a través de Análisis Factorial Confirmatorio (AFC). Como predictoras se han usado las sociodemográficas y la que no actuaba como variable criterio. El análisis completo ha permitido la comprobación del comportamiento de la muestra desde la perspectiva las variables sociodemográficas, así como la interacción entre las variables de estudio.

\section{Resultados}

Para la variable Miedo, la opción "poco miedo", con algo más de la mitad de la muestra $(50.4 \%)$ es la más frecuente, seguida de la de bastante miedo, alcanzada por el $31.2 \%$ de los adolescentes. Esto hace que las opciones centrales sean las que aglomeran porcentajes más altos, alejados de la opción más baja $(16.2 \%)$ y de mucho miedo $(2.2 \%)$.

Los resultados resumidos en la tabla 1 muestran que el Miedo Total está bajo la influencia de las variables Género, Habilidades Sociales y Cultura/Religión. El conjunto de ellas dan cuenta del $26 \%$ de la varianza en el Miedo $\left(\mathrm{R}^{2}=.260\right)$. El mayor poder determinante lo ejerce la variable Género, que por sí solo explica el $41.9 \%$ de la varianza $(\beta=.419)$. Esta variable, adopta valores positivos, lo que indica que son las chicas las que tienen niveles más altos de Miedo (dado que se asoció el valor 2 para el género femenino, mientras que fue de 1 para los varones). Lo mismo ocurre con la Cultura, teniendo niveles superiores los musulmanes (ocurre lo mismo, siendo este grupo cultural asociado al valor 2). Sin Tabla 1

Regresión miedo total

\begin{tabular}{|c|c|c|c|c|c|}
\hline$R=.509$ & $F=64.491$ & $p=.0000$ & & & \\
\hline \multicolumn{2}{|r|}{ Variables } & $B$ & $\beta$ & $t$ & $p$ \\
\hline \multirow{2}{*}{\multicolumn{2}{|c|}{$\begin{array}{c}\text { Constante General } \\
\text { Género }\end{array}$}} & 90.930 & & 7.355 & .000 \\
\hline & & 33.627 & .419 & 11.222 & .0000 \\
\hline \multirow{2}{*}{\multicolumn{2}{|c|}{$\begin{array}{c}\text { Habilidades Sociales } \\
\text { Cultura/Religión }\end{array}$}} & -.614 & -.209 & -5.608 & .000 \\
\hline & & 6.299 & .075 & 2.055 & .040 \\
\hline
\end{tabular}


Tabla 2

Regresión miedo animales

\begin{tabular}{cccccc|}
\hline $\mathbf{R}=\mathbf{. 5 5 2}$ & $\mathbf{R}^{2}=. \mathbf{3 0 5} \mathbf{F}=\mathbf{8 0 . 5 7 7}$ & $\mathbf{p =} . \mathbf{0 0 0 0}$ & $\boldsymbol{\beta}$ & $\boldsymbol{t}$ & $\boldsymbol{p}$ \\
\hline Variables & $\mathbf{B}$ & & 13.733 & .0000 \\
Constante General & 5.322 & .473 & 13.093 & .0000 \\
Género & 12.301 & .166 & .666 & .000 \\
Habilidades Sociales & 4.485 & -.144 & -3.989 & .000 \\
Cultura/Religión & -.137 & &
\end{tabular}

embargo, las Habilidades Sociales son inversamente proporcionales (aumentan sus niveles a medida que disminuyen los de Miedo). Si se sigue la función que aparece a continuación, la regresión hace posible calcular el Miedo Total, conocidas el resto de variables. Miedo Total $=90.241+33.627$ (Género) - .614 (HHSS) +6.299 (Cultura/Religión).

Los resultados de la regresión múltiple (Tabla 2) indican que el factor de Miedo a los Animales y al Peligro, viene determinado por las variables descritas en los totales: Género, Cultura/Religión y Habilidades Sociales. El conjunto de ellas da cuenta del $30.5 \%$ de la varianza en Miedo a los Animales y al Peligro $\left(\mathrm{R}^{2}=.305\right)$. El mayor poder determinante lo tiene, de nuevo, el Género, que explica el $47.3 \%$ de la varianza $(\beta=.473)$. Miedo Animales $=5.322+12.301$ (Género) +4.485 (Cultura/Religión) - .137 (HHSS).

La regresión arroja los datos (Tabla 3) que permiten ver como el Miedo a la Muerte también está bajo la influencia de las variables Género y Habilidades Sociales. Entre ambas suman el $16.5 \%$ de la varianza de este factor del Miedo $\left(\mathrm{R}^{2}=.165\right)$. La variable más determinante vuelve a ser el Género, que por sí sola explica el $36.2 \%$ de la varianza $(\beta=.362)$. El resto de relaciones han quedado rechazadas, incluyendo la
Cultura/Religión que aparecía tanto en los totales como en factores anteriores. Miedo Muerte $=44.878+11492$ (Género) -.151 (HHSS).

En la tabla 4 se reflejan los resultados que permiten demostrar que el factor de Miedo a lo Desconocido está bajo la influencia de las variables Género y Habilidades Sociales. El conjunto de ellas dan cuenta del $25.5 \%$ de la varianza en este factor de Miedo, $\left(\mathrm{R}^{2}=.255\right)$. El mayor poder determinante lo vuelve a ejercer el Género, que por sí solo explica $35.6 \%$ de la varianza $(\beta=.356)$. Las Habilidades Sociales vuelven a sr inversamente proporcionales a las puntuaciones en Miedo a lo Desconocido. Miedo Desconocido $=17.084+6.272$ (Género) - .164 (HHSS).

Los resultados de la regresión (Tabla 5) permiten predecir el factor Miedos Escolares si se conocen las puntuaciones en Habilidades Sociales y el valor asignado al Género. Todas ellas suman el $10 \%$ de la varianza en los Miedos Escolares $\left(\mathrm{R}^{2}=.100\right)$.

El mayor poder determinante lo vuelve a ejercer la puntuación en Habilidades Sociales, que explica el $22.7 \%$ de la varianza $(\beta=.227)$. Las Habilidades Sociales son inversamente proporcionales a este factor de Miedo. Las chicas obtienen niveles superiores de Miedo.

Tabla 3

Regresión miedo muerte

\begin{tabular}{cccccc|}
\hline $\mathbf{R}=. \mathbf{4 0 6}$ & $\mathbf{R}^{2=} . \mathbf{1 6 5} \mathbf{F}=\mathbf{5 4 . 5 7 7}$ & $\boldsymbol{p}=\mathbf{. 0 0 0 0}$ & $\boldsymbol{\beta}$ & $\boldsymbol{t}$ & $\boldsymbol{p}$ \\
\hline Variables & $\boldsymbol{B}$ & & 9.366 & .0000 \\
Constante General & 44.878 & .362 & 9.150 & .0000 \\
Género & 11.492 & -.130 & -3.281 & .001 \\
Habilidades Sociales & -.151 &
\end{tabular}


Tabla 4

Regresión miedo desconocido

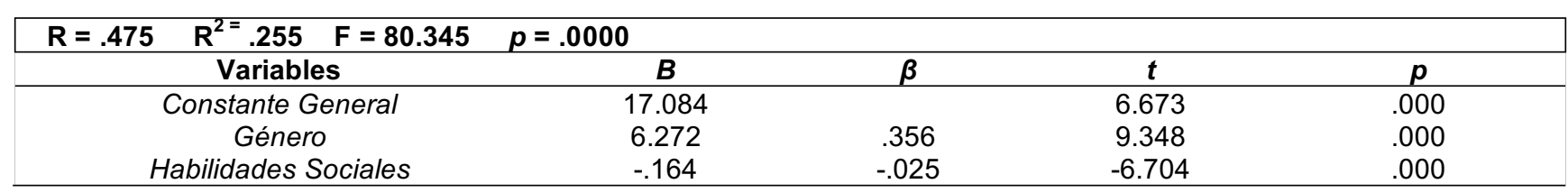

Miedos Escolares $=21.618-.125$ (HHSS) +2.768 (Género).

Para el factor de Miedos Médicos, los cálculos de la regresión reflejan que sus niveles se pueden anticipar si se conocen las variables Habilidades Sociales y Género. Todas las variables predictoras representan el $5.3 \%$ de la varianza en los Miedos Médicos $\left(\mathrm{R}^{2}=.053\right)$. El mayor poder determinante vuelve a tenerlo la puntuación en Habilidades Sociales, que explica el $15.1 \%$ de la varianza $(\beta=-.151)$. Existe una relación inversamente proporcional entre los Miedos Médicos y las Habilidades Sociales. La variable Género vuelve a adoptar un valor positivo. Los datos que han permitido realizar esta descripción aparecen resumidos en la Tabla 6. Miedos Médicos $=4.812-.037($ HHSS $)+.973$ (Género) .

Entre las variables predictoras, ocupando el primer lugar como la más influyente destaca el Género, siendo las chicas las que evidencian un nivel más alto de Miedo que los chicos. Esta primera perspectiva se ve reflejada en las medias de ambos (79.6 los varones y 116.7 las mujeres) y queda clara al considerar la estadística inferencial, como confirman las pruebas ANOVA y Chicuadrado de Pearson. Las diferencias son significativas en el en el Miedo Total $(p=.000)$, el Miedo a los Animales y al Peligro $(p=.000)$, a la Muerte $(p=.000)$, a lo Desconocido $(p=.000)$, los Miedos Escolares $(p=.000)$ y los Miedos Médicos $(p=.000)$. En todos ellos, el Género femenino refleja niveles más altos.

Por otra parte, entre las variables sociodemográficas, también la Cultura actúa como variable predictora, en los Totales y algunos factores de Miedo. El alumnado cristiano manifiesta niveles más bajos de miedo (su media es de 94.55 por 102.65). Los resultados de la estadística inferencial confirman que la cultura influye en el Miedo Total $(p=.022)$ y el Miedo a los Animales y al Peligro $(p=.000)$. Por otro lado, a través de este análisis se rechazaron para el Miedo a lo Desconocido $(p=.099)$, a la Muerte $(p=.781)$, los Miedos Escolares $(p=.052) \mathrm{y}$ los Miedos Médicos $(p=.231)$.En los casos positivos, los musulmanes reflejan niveles superiores de miedo.

Tabla 5

Regresión miedos escolares

\begin{tabular}{|cccccc|}
\hline $\mathbf{R}=. \mathbf{3 1 6}$ & $\mathbf{R}^{2=} . \mathbf{1 0 0} \mathbf{F}=\mathbf{3 0 . 6 9 2}$ & $\mathbf{p = . 0 0 0 0}$ & $\boldsymbol{\beta}$ & $\boldsymbol{t}$ & \\
\hline Variables & $\mathbf{B}$ & & $\mathbf{p}$ & .000 \\
Constante General & 21.618 & -.227 & -5.519 & .000 \\
Habilidades Sociales & -.125 & .183 & 4.455 & .000 \\
Género & 2.768 &
\end{tabular}


Tabla 6

Regresión miedos médicos

\begin{tabular}{|cccccc|}
\hline $\mathbf{R}=\mathbf{3 1 6}$ & $\mathbf{R}^{\mathbf{2}} \mathbf{. 1 0 0} \mathbf{F}=\mathbf{3 0 . 6 9 2}$ & $\mathbf{p = . 0 0 0 0}$ & $\boldsymbol{\beta}$ & $\boldsymbol{t}$ \\
\hline Variables & $\boldsymbol{B}$ & & $\mathbf{p}$ \\
\hline Constante General & 21.618 & -.227 & .000 \\
Habilidades Sociales & -.125 & .183 & .519 & .000 \\
Género & 2.768 & 4.455 & .000 \\
\hline
\end{tabular}

Con respecto a los niveles totales en la variable Habilidades Sociales, ninguno de los encuestados alcanzó la opción "muy pocas Habilidades Sociales". La opción bastante es la más frecuente, con un $72 \%$, seguida por la que refleja mucho $(23.7 \%)$. La opción minoritaria es la que se relaciona con niveles más bajos ("poco" con el $4.2 \%$ de la muestra).

Las Habilidades Sociales, tal y como se demuestra en la Tabla 7, está exclusivamente bajo la influencia de las puntuaciones en Miedo Total, dando cuenta del $8.2 \%$ de la varianza en Habilidades Sociales $\left(\mathrm{R}^{2}=.082\right)$. El poder determinante es del $28.6 \%$ de la varianza $(\beta=.286)$. Ambos factores vuelven a ser inversamente proporcionales, lo que indica que a más Miedo, menos Habilidades Sociales. La función permite calcular las puntuaciones si se conocen los niveles de Miedo. Habilidades Sociales $=96.802-.097$ (Miedo Total).

Los resultados resumidos en la Tabla 8 muestran que la manifestación de Disconformidad, como factor de las
Habilidades Sociales, está influido por el Miedo Total, de forma exclusiva, dando una varianza del $6.2 \%$ $\left(\mathrm{R}^{2}=.062\right)$. El poder determinante que ejerce esta variable es del $24.9 \%$ de la varianza $(\beta=.249)$. Existe una relación inversamente proporcional entre ambas variables. Disconformidad $=23.978-.031$ (Miedo Total).

De nuevo, las puntuaciones de Miedo Total, es la única variable que ejerce influencia en la manifestación de Enfado. El Miedo explica el $23.8 \%$ de la varianza total $(\beta=.238$ ), que es del $19.7 \%$ (si consideramos la $\mathrm{R}^{2}$ con un valor de .056). Las variables vuelven a ser inversamente proporcionales. El resto de variables quedan rechazadas. Los resultados quedan resumidos en la Tabla número 9. Enfado=28.428 - .030 (Miedo Total).

Tabla 7

Regresión Habilidades Sociales

\begin{tabular}{|c|c|c|c|c|}
\hline $\mathrm{R}^{2=} .082 \quad \mathrm{~F}=49.223$ & $p=.0000$ & & & \\
\hline Variables & B & $\beta$ & $\boldsymbol{t}$ & $p$ \\
\hline Constante General & 96.802 & & 65.020 & .000 \\
\hline Miedo Total & -.097 & -.286 & -7.016 & .000 \\
\hline
\end{tabular}


Tabla 8

Regresión disconformidad

\begin{tabular}{|cccccc|}
\hline $\mathbf{R}=.249$ & $\mathbf{R}^{2=} \mathbf{. 0 6 2} \mathbf{F}=\mathbf{3 6 . 6 1 4}$ & $\boldsymbol{p}=. \mathbf{0 0 0 0}$ & $\boldsymbol{\beta}$ & $\boldsymbol{t}$ & $\boldsymbol{p}$ \\
\hline Variables & $\boldsymbol{B}$ & & 42.926 & .0000 \\
& Constante General & 23.978 & -.249 & -6.051 & .000 \\
Miedo Total & -.031 & & & \\
\hline
\end{tabular}

En la tabla 10 se reflejan los resultados que demuestran que la Interacción con personas del Sexo Opuesto, factor de las Habilidades Sociales, se puede predecir conocido el Género al que pertenece el sujeto. Esta es la única variable influyente, quedando rechazadas el resto, incluyendo las puntuaciones de Miedo Total. La varianza total es del $2.2 \%\left(\mathrm{R}^{2}=.022\right)$, explicando el Género el $14.8 \% \quad(\beta=.148)$. Las puntuaciones más altas se dan entre los varones (asociado al valor 1), obteniendo un valor negativo en este sentido. La función que se observa a continuación permite averiguar la puntuación en este factor, si se conoce el género del sujeto. Interacción Sexo Opuesto $=11.796-.898$ (Género).

Los datos puestos de manifiesto por la regresión múltiple (Tabla 11) indican que la manifestación de Opiniones está bajo la influencia del Miedo Total así como la Cultura/Religión. Entre ambas dan cuenta del $5.6 \%$ de la varianza en este factor $\left(\mathrm{R}^{2}=.056\right)$. El mayor poder determinante vuelve a ejercerlo la puntuación en Miedo Total, que explica el $19.9 \%$ de la varianza $(\beta=.199)$. Ambas variables son inversamente proporcionales. Por su parte, la Cultura toma un valor positivo, lo que indica que las puntuaciones son mayores entre los musulmanes (que se asocian al valor 2). Para calcular este factor se puede seguir la función de regresión que aparece a continuación. Opiniones $=13.143 \quad-\quad .015 \quad($ Miedo $\quad$ Total $) \quad+.908$ (Cultura/Religión).

Finalmente, para la Asertividad, los resultados de la regresión múltiple (Tabla 12) indican que este factor está también bajo la influencia del Miedo Total, siendo ambas variables inversamente proporcionales. La varianza es del $2.8 \%\left(\mathrm{R}^{2}=.028\right)$, representando el Miedo Total $16.6 \%$ de la varianza $(\beta=.166)$. Asertividad $=12.708-.011$ (Miedo Total).

Entre los predictores de las Habilidades Sociales, dentro de las variables sociodemográficas, aparece el Género, aunque está sólo un uno de los factores. En cualquier caso, los chicos tienen un nivel ligeramente superior a las chicas, tal y como reflejan las medias de ambos (89.9 varones y 84.8 chicas). Todo ello indica la dirección de las diferencias estadísticamente significativas, encontradas en Habilidades Sociales Totales $(p=.000)$, expresión de Enfado $(p=.027)$, Asertividad $(p=.006)$, Disconformidad $(p=.000) \mathrm{e}$ Interacción con el Sexo opuesto $(p=.000)$. El factor "realización de Peticiones" ( $p=.509)$ fue el único en el que no fueron significativas.

Tabla 9

Regresión enfado

\begin{tabular}{|c|c|c|c|c|c|}
\hline $\mathrm{R}=.238$ & $R^{2}=.056 \quad F=33.153$ & $p=.0000$ & & & \\
\hline \multicolumn{2}{|r|}{ Variables } & $B$ & $\beta$ & $\bar{t}$ & $p$ \\
\hline \multicolumn{2}{|r|}{ Constante General } & 28.428 & & 49.997 & .0000 \\
\hline \multicolumn{2}{|r|}{ Miedo Total } & -.030 & -.238 & -5.758 & .000 \\
\hline
\end{tabular}


Tabla 10

Regresión interacción sexo opuesto

\begin{tabular}{cccccc|}
\hline $\mathbf{R}=.148$ & $\mathbf{R}^{2=} . \mathbf{0 2 2} \mathbf{F}=\mathbf{1 2 . 3 7 7}$ & $\boldsymbol{p}=. \mathbf{0 0 0 0}$ & $\boldsymbol{\beta}$ & $\boldsymbol{t}$ & $\boldsymbol{p}$ \\
\hline Variables & $\boldsymbol{B}$ & & 28.527 & .0000 \\
& Constante General & 11.796 & -3.518 & .000 \\
Género & -.898 & -.148 & - & \\
\hline
\end{tabular}

Con la variable Cultura/Religión ocurre exactamente lo mismo: aparece como predictor sólo en un factor. Considerando el grupo cultural de referencia, sólo se puede destacar una enorme igualdad, ya que cristianos y musulmanes tienen una media idéntica (87.11).Esto indica que las diferencias no fueron significativas en las Habilidades Sociales Totales ( $p=.999)$, la Disconformidad $(p=.065)$, la expresión de Enfado $(p=.296)$, la Interacción con personas del Sexo opuesto $(p=.183)$, la Asertividad $(p=.663)$ y la realización de Peticiones $(p=.750)$. Sin embargo, en el factor de manifestación de Opiniones (en la que actúa como predictor) sí se obtuvieron diferencias significativas $(p=.002)$.

Se ha dejado para el final el análisis de la interacción entre ambas variables. En este sentido, la interacción parece clara, dado que actúan como predictores importantes de manera recíproca. De esta manera, las Habilidades Sociales aumentan a medida que disminuye el nivel de miedo. Así, los niveles más altos se dan entre el alumnado que presenta menos miedo (92.31), recogiéndose los mayores porcentajes en mucho (38.9\%) y los menores en poco (3.3\%). Después vendría el grupo que refleja poco miedo (88.47), con los segundos porcentajes en la opción "muchas Habilidades Sociales" $(25.6 \%)$, seguido por los que manifiestan bastante (82.99). Los que más miedo reflejan son los que menos Habilidades Sociales declaran con los porcentajes más bajos en mucho (0\%) y los mayores en poco (16.7\%). Considerando la prueba ANOVA, aparecen diferencias estadísticamente significativas en las Habilidades Sociales $(p=.000)$, la Disconformidad $(p=.000)$, la expresión de Enfado $(p=.000)$, la Asertividad $(p=.005)$ y la manifestación de Opiniones $(p=.000)$. Por el contrario, no fueron significativas en la Interacción con el Sexo opuesto $(p=.054)$ y la realización de Peticiones $(p=.782)$. En ambos casos, el Miedo no actúa como predictor.

Tabla 11

Regresión opinioines

\begin{tabular}{|cccccc|}
\hline $\mathbf{R}=. \mathbf{2 3 7}$ & $\mathbf{R}^{2=} . \mathbf{0 5 6} \mathbf{F}=\mathbf{1 6 . 3 9 8}$ & $\boldsymbol{p}=. \mathbf{0 0 0 0}$ & $\boldsymbol{\beta}$ & $\boldsymbol{t}$ \\
\hline Variables & $\mathbf{B}$ & & 25.984 & .0000 \\
Constante General & 13.143 & -.199 & -4.789 & .000 \\
Miedo total & -.015 & .149 & 3.580 & .000 \\
Cultura/Religión & .908 & & \\
\hline
\end{tabular}


Tabla 12

Regresión asertividad

\begin{tabular}{|cccccc|}
\hline $\mathbf{R}=.166$ & $\mathbf{R}^{2=} . \mathbf{0 2 8}$ & $\mathbf{F}=\mathbf{1 5 . 7 0 1}$ & $\boldsymbol{p}=\mathbf{. 0 0 0 0}$ & $\boldsymbol{\beta}$ & $\boldsymbol{t}$ \\
\hline Variables & $\boldsymbol{B}$ & & 43.189 & .0000 \\
\hline Constante General & 12.708 & -.166 & -3.963 & .000 \\
Miedo total & -.011 & & \\
\hline
\end{tabular}

\section{Discusión/Conclusiones}

La población estudiada refleja niveles medio-bajos de miedo, siendo el factor que genera más miedo el relacionado con la muerte y menos los miedos médicos. El Género es el factor más determinante entre los predictores, dado que aparece en todos los casos. Ocurre lo mismo con la Cultura, a pesar de que no aparece en la mayoría de factores (sí en los Totales). Con respecto a la primera variable, en todos los casos, las mujeres reflejan niveles más altos. Esta realidad era algo que se esperaba, teniendo en cuenta los trabajos de Kushnir et al. (2014), Miloyan et al. (2014) y Pulido y Herrera (2015). Esta realidad puede relacionarse con la educación diferencial que reciben niños y niñas, correspondiente con los diferentes estilos de crianza de ambos (Méndez, Inglés, Hidalgo, García-Fernández y Quiles, 2003), lo que justificaría el pensamiento tradicional de que las chicas tengan un mayor repertorio emocional. Por su parte, la cultura también determina las manifestaciones de Miedo, siendo los musulmanes los que presentan niveles superiores para esta variable. Diferencias para los grupos culturales también se encontraron en Roth et al. (2014) y Pulido y Herrera (2015). La población musulmana presenta una procedencia marroquí con un altísimo nivel de analfabetismo y una elevada natalidad, así como un estatus socioeconómico y cultural bajo, serios problemas de enculturación y bilingüismo (integración por la comunicación) y con una presencia muy marcada de su religión, el islam, en sus vidas (Herrera, 2000). Esta realidad justifica las dificultades para la integración socio educativa (Martínez y Alfageme, 2004). Estas diferencias en los núcleos familiares de ambos grupos culturales (Méndez et al., 2003), con estilos de crianza distintos, unido a las diferencias en la lengua materna (Roa, 2006) entre ambas etnias, contribuyen a la aparición de problemas de integración socioemocional del alumnado no autóctono. El contexto "autóctono" se mostraría como un impulso para el desarrollo socioafectivo (Pulido y Herrera, 2015). No aparecen diferencias estadísticamente significativas en cuanto al estatus, a pesar de que las dos últimas variables se encuentran íntimamente relacionadas entre sí. Los resultados son contrarios a los encontrados en otras investigaciones, en las que sí existían diferencias en los niveles de miedo por el estatus: Kushnir et al. (2014) y Roth et al. (2014), en los que se demuestra la influencia del entorno socio-cultural sobre el miedo. No existen diferencias visibles en los niveles de miedo tampoco por edad, apareciendo gran igualdad en la etapa adolescente (Miloyan et al., 2014). Las dos últimas variables tampoco actúan como predictoras.

La población refleja resultados medio-altos en la categoría de Habilidades Sociales. Entre las variables sociodemográficas sólo el Género y la Cultura actúan como predictores y, además sólo aparecen en un factor. La variable Género no es un factor determinante, dado que aparece sólo en varones que tienen resultados significativamente superiores a las mujeres. Esta realidad resulta contraria a las conclusiones de Gomes y Pereira (2014), donde se determina que son las niñas las que obtienen mejores resultados. Esta realidad se debe, probablemente, a la muestra del mencionado trabajo, con una población exclusivamente infantil. La Cultura tampoco ejerce una influencia clara en las puntuaciones de Habilidades Sociales, a pesar de que funciona como predictor en uno de los factores que la conforman. La cultura trae consigo numerosas diferencias en los 
patrones educativos y los valores transmitidos a los más pequeños, algo recogido en el trabajo de Braz et al. (2013), donde los datos mostraron evidencia de la transmisión intergeneracional de varias clases de habilidades sociales. Con respecto a la edad, no se contemplan diferencias, algo que resulta contrario a lo comentados en Gomes y Pereira (2014), donde se contempla un aumento progresivo a medida que aumenta la edad. A edades tan tempranas, las niñas se caracterizan por tener una mayor madurez a los niños, algo que justificaría las diferencias encontradas en esta etapa evolutiva. Por otra parte, el estatus tampoco juega un papel preponderante, a pesar de los datos sacados del estudio de Alonso y Román, (2014), en el que el nivel económico y sociocultural correlaciona positivamente con el estilo educativo familiar, y éste, a su vez, con el nivel de autoestima mayor que repercute en diferentes dimensiones de desarrollo: ajuste emocional, éxito social y académico.

Finalmente, las Habilidades Sociales son inversamente proporcionales a los niveles de miedo manifestados por el sujeto, algo esperado, teniendo en cuenta trabajos como los de Pulido y Herrera (2015). Además se comportan como uno de los predictores más importantes de forma recíproca. En el caso del Miedo, es el predictor más determinante para los totales y cada uno de la mayoría de los factores de las Habilidades Sociales. Esta realidad no es difícil de entender si se considera que las personas que evidencian mayores niveles de miedo son más reacias a la interacción y el contacto con los demás. Del mismo modo, la interacción con otras personas permite compartir experiencias, así como adquirir aprendizajes de otras personas, lo que crea una mayor "apertura de mente", una mayor posibilidad de contrastar las experiencias negativas y positivas con los demás, lo que llegaría a reducir el miedo que la persona pudiera llegar a manifestar, por la mayor seguridad que da ese intercambio con iguales.

En cuanto a las implicaciones prácticas socioeducativas, es fundamental la implementación de programas de educación emocional, posibilitando una intervención más eficaz con efectos positivos en el desarrollo personal de los adolescentes. Para ello se recomiendan las escuelas de padres o programas de educación emocional, permitiendo que se desarrollen conductas positivas y relaciones sociales satisfactorias (Ramírez-Lucas, Ferrando y Sáinz, 2015).

Para finalizar se comentan las limitaciones encontradas en el presente trabajo como son la distribución de la muestra por edades, así como el tamaño de las submuestras culturales, lo cual es fruto de centrar este trabajo en un contexto limitado (como es la ciudad autónoma de Ceuta). Las diferencias encontradas en este contexto hacen aconsejable considerar de manera más profunda algunos elementos fundamentales de la multiculturalidad, como pueden ser el estilo de crianza, la manera en la que viven su religión o las diferencias lingüísticas dentro del entorno socio-familiar seguidos por cada uno de los grupos culturales. Dichas limitaciones pueden ser la base de futuras investigaciones dentro del ámbito, considerando el entorno multicultural como un ámbito a explorar dentro del presente y de otros campos de estudio.

\section{Referencias}

Alonso, J. y Román, J. M. (2014). Nivel sociocultural, prácticas educativas familiares y autoestima de los hijos en edades tempranas. Revista de Investigación Educativa,32(1), 187-202.

Ascensio, M., Vila, M. G., Robles-García, R., Páez, F., Fresán, A. y Vázquez, L. (2012). Estudio de traducción, adaptación y evaluación psicométrica del Inventario de Miedos FSSC-II en una muestra de estudiantes de educación media superior. Salud Mental, 35, 195-203.

Braz, A. C., Cômodo, C. N., Del Prette, Z., Del Prette, A. y Fontaine, A. (2013). Habilidades sociales e intergeneracionalidad en las relaciones familiares. Apuntes de Psicología, 31(1), 77-84.

Bueno, M. R., Durán, M. y Garrido, T. (2013). Entrenamiento en habilidades sociales en estudiantes 
universitarios de Magisterio de la especialidad de Educación Primaria: un estudio preliminar. Apuntes de Psicología, 31(1), 85-91.

Delgado, B., Inglés, C. J. y García, J. M. (2014). Ansiedad social subclínica y rendimiento académico en la adolescencia: análisis de sus implicaciones teórico-prácticas. Estudios de Psicología, 31(1), 58-79.

Ford, B., Mauss, I., Troy, A., Smolen, A. y Hankin, B. (2014). Emotion Regulation Moderates the Risk Associated with the 5-HTT Gene and Stress in Children. Emotion, 14(5), 930-939

Gismero E. (2000). EHS, Escala de Habilidades Sociales. Madrid, España: TEA Publicaciones de Psicología Aplicada.

Gomes, R. M. y Pereira, A. (2014). Influence of Age and Gender in Acquiring Social Skills in Portuguese Preschool Education. Psychology. 5(2), 99-103.

Herrera, F. (2000). La inmigración extranjera no comunitaria y la convivencia en contextos concretos: el caso de Ceuta. En Instituto de Estudios Ceuties, Monografía de los cursos de Verano de la Universidad de Granada en Ceuta (12 ed., pp. 357-359). Ceuta, España: Instituto de Estudios Ceutíes-Universidad de Granada.

Kushnir, J., Gothelf, D. y Sadeh, A. (2014). Nighttime fears of preschool children: A potential disposition marker for anxiety? Comprehensive Psychiatry, 55, 336-341.

Martínez, M. y Alfageme, A. (2004). Integración socioeducativa del alumno gitano en la escuela española. Revista Española de Educación Comparada. 10, 299-324.

Matesanz, A. (2006). Datos para la adaptación castellana de la Escala de Temores (FSS). Análisis $y$ Modificación de Conducta, 32(144), 521-551.
Méndez, F. X., Inglés, C. J., Hidalgo, M. D., GarcíaFernández, J. M. y Quiles, M. J. (2003). Los miedos en la infancia y la adolescencia: un estudio descriptivo. Revista Electrónica de Motivación y Emoción, 6, 13-25. Recuperado de http://reme.uji.es.

Miloyan, B., Bulley, A., Pachana, N. y Byrne, G. (2014). Social Phobia symptoms across the adult lifespan. Journal of Affective Disorders, 168, 86-90.

Pulido, F. y Herrera, F. (2015). Miedo e inteligencia emocional en el contexto pluricultural de Ceuta. Anuario de Psicología, 45(2), 249-263.

Ramírez-Lucas, A., Ferrando, M. y Sáinz, M. (2015). ¿Influyen los estilos parentales y la Inteligencia Emocional de los padres en el desarrollo emocional de sus hijos escolarizados en $2^{\circ}$ ciclo de Educación Infantil? Acción Psicológica, 12(1), 65-78. http://dx.doi.org/10.5944/ap.12.1.14314

Roa, J. M. (2006). Rendimiento escolar y "situación diglósica" en una muestra de escolares de educación primaria en Ceuta. Revista Electrónica de Investigación Educativa, 8, 1-15.

Roth, G., Benita, M. Amrani, C., Shachar, B. Asoulin, H., Moed, A., Bibi, U. y Kanat-Maymon, Y. (2014). Integration of negative emotional experience versus suppression: Addressing the question of adaptive functioning. Emotion, 14(5), 908-919.

Tobías-Imbernón, C., Olivares-Olivares, P. J. y Olivares, J. (2013). Revisión de autoinformes de fobia social diseñados para niños y adolescentes. Revista Mexicana de Psicología, 30(2), 123-130. 